\title{
Asfixia Perinatal e Problemas Cardíacos
}

\author{
Gesmar Volga H. Herdy, Vânia Glória S. Lopes, Maria Luiza S. Aragão, Carlos Alberto M. Pinto, Plínio \\ A. Tavares Jr., Fabiano B. Azeredo, Pablo M. C. Nascimento
}

Niterói, RJ

Objetivo - Avaliar a gravidade das complicações cardíacas na asfixia neonatal, sua evolução e correlacionálas com o grau e duração do processo hipóxico.

Métodos - Foram estudados 90 bebês nos últimos 7 anos com grau de Apgar $\leq 6$ no $5^{\circ}$ min de vida. Pelo protocolo, após o exame físico e os cuidados intensivos, eram submetidos a dosagem do pH arterial, CPK-MB, DHL, glicemia, além da realização de radiografia de tórax, eletrocardiograma (ECG), ecocardiograma, seriados e repetidos a cada semana. Aqueles que faleceram eram levados à necropsia.

Resultados - Dos 90, 73 (81\%) eram prematuros, 30 (41\%) eram apropriados para a idade gestacional ( $A I G)$ e 43 (59\%) eram pequenos(PIG). Em 21 (23\%) casos havia $\mathrm{pH}$ arterial $<7,2$. Os quadros clínicos mais observados foram: pneumonia em 28 (31\%), anemia 24 (26\%) e icterícia moderada $12(5 \%)$, entre outros. Ao exame fisico observaram-se: sopro sistólico em $46(50 \%)$, ictus propulsivo $18(20 \%)$ e ICC em $8(9 \%)$. No ECG, os sinais mais freqüentes foram alterações de repolarização (STe T) em 44 (49\%). No ecocardiograma, observou-se persistência do canal arterial (PCA) em 20 (22\%), regurgitação tricúspide em $6(7 \%)$, hipertensão pulmonar em $6(8 \%)$, hipocontratilidade de VE e dilatação de VD em 4 (5\%). Dos 23 óbitos, 14 foram estudados e as alterações mais freqüentes foram necrose de fibras em 8 (68\%) casos e em 4 (29\%) congestão, vacuolização e perda de estriação.

Conclusão - A maioria teve evolução favorável mesmo naqueles que tiveram acidemia importante. Muitas alterações de ECG e ecocardiograma se normalizaram. Daqueles que evoluíram para o óbito, as lesões mais graves ocorreram nos que sofreram, por mais tempo, processo anóxico.

Palavras-chave: asfixia perinatal, coração

\section{Perinatal Asphyxia and Heart Problems}

Purpose - To evaluate the severity of cardiac complications of neonatal asphyxia in relation to the length and degree of hipoxia.

Methods - Ninety babies with an Apgar score $\leq 6$ were examined in the intensive care unit at our institution during seven years. Arterial blood for measuring $\mathrm{pH}$, glucose, $L D H$ and $M B$ fraction of $C K$, together with serial electrocardiogram (ECG), echocardiogram and chest $X$ rays was obtained. The fatal cases were studied with macro and microscopic examination.

Results - From a total of 90 cases, 73 were premature: $30(41 \%)$ appropriate for gestation age (AGA) and $43(59 \%)$ small for gestation age (SGA). Twenty one $(23 \%)$ cases had arterial $p H<7.2$. The most common clinical conditions were: pneumonia 28 (31\%), anemia 24 (26\%) and jaundice $12(13 \%)$. The main cardiological findings were: systolic murmur in $46(50 \%)$, signs of heart hypertrophy in $18(20 \%)$ and heart failure in $8(9 \%)$. On ECG the main findings were ST and T abnormalities. The echocardiogram showed a patent ductus arteriosus (PDA) in $20(22 \%)$, tricuspid regurgitation in $6(7 \%)$, pulmonary hypertension in $6(7 \%)$, dyskinesia and ventricular dilatation in $4(5 \%)$. Necropsy was performed in 23 cases and macro and microscopy obtained in 14; the most frequent findings were: myocite necrosis in $8(54 \%)$, congestion, vacuolization and loss of striae in $4(29 \%)$.

Conclusion - In the majority of cases, patients had a benign course, even those presenting with severe acidemia. Many abnormal EKGs and echocardiograms became normal after a few weeks. Among those who had a fatal outcome, the severity of histological lesions was observed in babies who had suffered asphyxia for more prolonged periods.

Key-words: perinatal asphyxia, heart
Hospital Universitário Antônio Pedro - Niterói, RJ

Correspondência: Gesmar Volga H. Herdy - Trav. Antônio Pedro, 10/301 - 24230030 - Niterói, RJ

Recebido para publicação em 12/2/98

Aceito em 21/5/98
A hipoxemia perinatal pode levar a várias alterações sistêmicas que variam desde graus leves e imperceptíveis até quadros graves e fatais. Em trabalhos anteriores, foram descritas complicações cardíacas da asfixia perinatal ${ }^{1,2}$. 
Foram observados alguns casos fatais por infarto extenso do miocárdio, sendo que a maioria das alterações encontradas era benigna e transitória. Além do miocárdio, a válvula tricúspide era freqüentemente atingida nesses casos. Segundo Freyman e col, regurgitação tricúspide ocorre por hipertensão pulmonar, que se segue à vasoconstrição da hipoxemia ${ }^{3}$. O objetivo deste estudo prospectivo foi avaliar o grau de comprometimento cardíaco, sua evolução correlacionando-o com o grau e duração do processo anóxico.

\section{Métodos}

Foram estudados, prospectivamente, 90 bebês de abril/90 a março/97, nascidos na maternidade do HUAP e considerados asfíxicos.

\begin{tabular}{|lcc|}
\hline \multicolumn{3}{|c|}{ Tabela I - Quadros clínicos dos pacientes } \\
\hline Quadros clínicos * & Número de casos & $\%$ \\
\hline Pneumonia & 28 & 31 \\
Anemia & 24 & 26 \\
Icterícia moderada & 12 & 13 \\
Septicemia & 10 & 11 \\
Tremores ou convulsões & 8 & 9 \\
Hipoglicemia & 6 & 7 \\
Sinais de baixa perfusão & 6 & 7 \\
Taquipnéia transitória & 5 & 6 \\
Enterocolite & 4 & 5 \\
Membrana hialina & 4 & 5 \\
Pneumotórax hipertensivo & 4 & 5 \\
Atresia de coana & 1 & 1 \\
\hline * Houve concomitância de um ou mais em alguns casos. \\
\hline
\end{tabular}

\begin{tabular}{|lcc|}
\hline \multicolumn{2}{|c|}{ Tabela II - Dados mais freqüentes ao exame cardiovascular } \\
\hline Sinais cardiovasculares * & Número de casos & $\%$ \\
\hline Sopro suave em bordo esternal esquerdo & 24 & 26 \\
Sopro sistólico na base & 22 & 24 \\
Ictus propulsivo & 18 & 20 \\
Pulsos amplos & 18 & 20 \\
Taquicardia(mais de 140bpm) & 16 & 18 \\
Insuficiência cardíaca congestiva & 8 & 9 \\
Hiperfonese de 2 ${ }^{\text {a }}$ bulha & 6 & 7 \\
Ritmo de galope & 4 & 5 \\
Arritmia & 4 & 5 \\
Sopro diastólico no bordo esternal esquerdo & 2 & 2 \\
\hline \multirow{2}{*}{ Houve concomitancia de um ou mais sinais em alguns casos. } \\
\hline
\end{tabular}

\begin{tabular}{|lcc|}
\hline \multicolumn{3}{|c|}{ Tabela III - Resultados obtidos ao eletrocardiograma } \\
\hline Resultados ECG & Número de casos & $\%$ \\
\hline Normal & 33 & 37 \\
Alt.leve repol.ventr. & 27 & 30 \\
Alt. import. repol. ventric. & 17 & 19 \\
Hipertrofia de VD & 5 & 6 \\
Hipertrofia de VE & 4 & 4 \\
Extra-sistolia supra V & 3 & 3 \\
Extra-sistolia ventricular & 1 & 1 \\
\hline Total & 90 & 100 \\
\hline
\end{tabular}

\begin{tabular}{|lcc|}
\hline \multicolumn{3}{|c|}{ Tabela IV - Resultados do ecocardiograma } \\
\hline Resultados do ecocardiograma & Número de casos & $\%$ \\
\hline Normal & 39 & 43 \\
PCA isolado & 20 & 22 \\
Regurgitação tricúspide & 6 & 7 \\
Hipertensão pulmonar & 6 & 7 \\
Hipocontrat.VE + dilatação VD & 4 & 5 \\
Líquido pericárdico & 4 & 5 \\
Hipertrofia de VD & 3 & 3 \\
CIV & 2 & 2 \\
PCA + CIA & 2 & 2 \\
Persistência de forame ovale & 2 & 2 \\
Hipoplasia Ao e VE & 1 & 1 \\
Tetralogia de Fallot & 1 & 100 \\
\hline Total & 90 & \\
\hline PCA- persistência do canal arterial; VE- ventrículo esquerdo; VD- ventrículo \\
direito; CIV- comunicação interventricular; CIA- comunicação interatrial; Ao- \\
aorta.
\end{tabular}

Como critério de inclusão para asfixia, adotamos grau Apgar $\leq 6$ no $5^{\circ} \mathrm{min}$, tendo sido excluídos os que não puderam ser investigados por problemas técnicos.

Segundo o protocolo, após os primeiros cuidados, os recém-nascidos eram submetidos à colheita de sangue para: gasometria arterial, glicemia, hemograma, VDRL, dosagem de cálcio, desidrogenase láctica (DHL), creatinofosfoquinase (CPK) e CPK-MB, com dosagens feitas nas primeiras $24 \mathrm{~h}$. Também eram realizados eletrocardiograma (ECG), ecocardiograma e radiografia de tórax nos primeiros dois dias de vida. O ecocardiógrafo utilizado foi color SIM 7000, nos últimos dois anos, e anteriormente um SIM 5000.

Dentre os 90 casos, 23 (25\%) evoluíram para o óbito no berçário e 14 foram necropsiados com estudo macro e microscópico de todos os órgãos.

\begin{tabular}{|c|c|c|c|c|c|}
\hline \multicolumn{6}{|c|}{ Tabela V - Estudo anatomopatológico em 14 casos } \\
\hline Dados de macroscopia & $\mathrm{N}^{\circ}$ Casos & $\%$ & Dados de microscopia & $\mathrm{N}^{\circ}$ Casos & $\%$ \\
\hline Congestão de Vasos do Coração & 5 & 37 & $\begin{array}{l}\text { Congestão vascular, } \\
\text { Vacuolização de fibras edema intersticial }\end{array}$ & 4 & 29 \\
\hline Petequias no epicardio & 2 & 14 & & & \\
\hline Persist. canal arterial & 2 & 14 & Áreas de necrose focal, miocardio & 3 & 21 \\
\hline Normal & 2 & 14 & Áreas de necrose e infiltrado monocleares & 3 & 21 \\
\hline Vegetações pequenas no endocárdio & 1 & 7 & Áreas extensas de necrose e calcificação & 2 & 14 \\
\hline Ruptura de músc. Papilar da Válvula tricúspide & 1 & 7 & $\begin{array}{l}\text { Infiltrado inflamatório polimorfos } \\
\text { (endocardite bacteriana) }\end{array}$ & 1 & 7,5 \\
\hline Petequias epicardio e aumento do coração & 1 & 7 & Necrose + Infiltrado monoclear por CMV & 1 & 7,5 \\
\hline Total & 14 & 100 & Total & 14 & 100 \\
\hline
\end{tabular}




\section{Resultados}

Dentre os 90 bebês, $73(81 \%)$ eram prematuros, sendo que destes, $30(41 \%)$ eram apropriados para a idade gestacional (AIG) e $43(59 \%$ ) pequenos (PIG). Dentre os 17 (19\%) a termo, 10 eram AIG e os demais PIG. Entre os prematuros, a idade mínima gestacional foi 28 semanas, a máxima 35 semanas e três dias, e a mediana 30 semanas e quatro dias. As gestações de risco somaram $38(42 \%)$, principalmente, por hipertensão materna, infecção, colagenose e diabetes. Ocorreram $43(47 \%)$ partos normais e $47(53 \%)$ cesarianas. Necessitaram manobras de ou reanimação 42 (46\%) bebês, sendo que 25 (27\%) permaneceram mais de dois dias com ventilação mecânica.

Foram observados níveis baixos de $\mathrm{pH}$ arterial $(<7,35)$ em $62(69 \%)$ casos sendo que em $21(23 \%)$ foi $<7,2$. A avaliação das dosagens das enzimas mostrou que alguns casos com DHL muito elevado não apresentavam gravidade clínica e vice-versa. Apenas em quatro recém-nascidos, com alterações de repolarização ventricular ao ECG, ocorreram níveis elevados de DHL. Altas taxas de CPK MB, em relação ao CPK total, foram mais fidedignas, pois ocorreram em bebês com comprometimento cardíaco mais importante, mas não foram dosadas em todos os casos.

Durante a permanência no berçário, foram observados os quadros clínicos contidos na tabela I e os dados mais freqüentes, encontrados no exame cardiovascular, na tabela II. Em 28 casos, havia também aumento da área cardíaca na radiografia de tórax (fig. 1).

O resultado do ECG, realizado nas primeiras $48 \mathrm{~h}$ de vida, foi normal em 33 (37\%), mostrou alterações leves de repolarização ventricular (achatamento de ondas T) em 27 (30\%), distúrbio acentuado de repolarização com ondas T negativas em 17 (19\%), hipertrofia de ventrículo direito(VD) em 5 (6\%), hipertrofia de ventrículo esquerdo em 4 (4\%), extra-sistolia supraventricular em $3(3 \%)$ e ventricular em 1 (1\%) (tab. III). Na figura 2 apresentamos um ECG com alterações importantes.

O ecocardiograma com Doppler foi normal em 39(43\%) casos, 22 (24\%) apresentaram sinais de persistência do canal arterial (PCA) e, destes, em dois havia associação com comunicação interatrial. Observamos regurgitação tricúspide em $6(7 \%)$, sendo em um muito grave, tendo evoluído com insuficiência cardíaca congestiva (ICC) refratária, provocada por infarto extenso do músculo papilar. Dentre os 22 casos com PCA, 10 eram prematuros e oito necessitando de respiração assistida. Outros achados encontram-se na tabela IV.

Quanto à evolução, 23 (26\%) bebês faleceram e os demais receberam alta para acompanhamento ambulatorial. A permanência no berçário foi em média de 28 dias $( \pm 10)$. Em alguns casos, foram observadas intercorrências importantes, como septicemia em 10, sendo que em dois por Klebsiella. Dois bebês tiveram hidrocefalia e um sinal de hemorragia ventricular. Quatro apresentaram enterocolite necrotizante e quatro pneumotórax hipertensivo. Todos estes casos necessitaram dos procedimentos cirúrgicos pertinentes.
Na figura 3 mostramos um exemplo das alterações histológicas.

Em 36 (40\%) bebês, foram obtidos ecocardiogramas após duas semanas: dos 22 que apresentavam PCA; em fecharam espontaneamente, quatro com indometacina, seis com procedimento cirúrgico e dois evoluíram para óbito. Os outros casos não eram hemodinamicamente importantes e tiveram alta para controle ambulatorial. Daqueles com regurgitação tricúspide no primeiro ecocardiograma, em quatro esta normalizou-se e um bebê teve alta ainda com esse quadro. O caso mais grave mostrou sinais de ruptura de cordoalha da válvula tricúspide, com ICC grave, tendo evolução fatal (fig. 4).

O ECG de controle foi feito em 25 (28\%) casos: os que apresentaram distúrbio de repolarização leve e três dos que eram mais graves normalizaram-se antes da alta. Os dois com extra-sistolia mantiveram-se assim, mesmo após a alta.

Dos 23 (26\%) que evoluíram para óbito, obtivemos estudo anatomopatológico completo em 14 casos, sendo que nove viveram menos de cinco dias (tab. V). Observamos quatro casos com congestão vascular, vacuolização e perda de estriação das fibras. Em oito bebês havia necrose dos miócitos, em três estas áreas eram focais e em dois eram extensas com calcificação. Em um, havia sinais de endocardite e, em outro, de miocardite com células de inclusão citomegálica.

Ao correlacionarmos a gravidade das lesões histológicas encontradas e o tempo de asfixia, observamos que havia descrição de eliminação de mecônio antes do parto (líquido amniótico verde e espesso) em sete casos, com menos de cinco dias de vida, onde em cinco havia edema intersticial, perda de estriação das fibras ou vacuolização e, em dois, necrose extensa e áreas de calcificação.

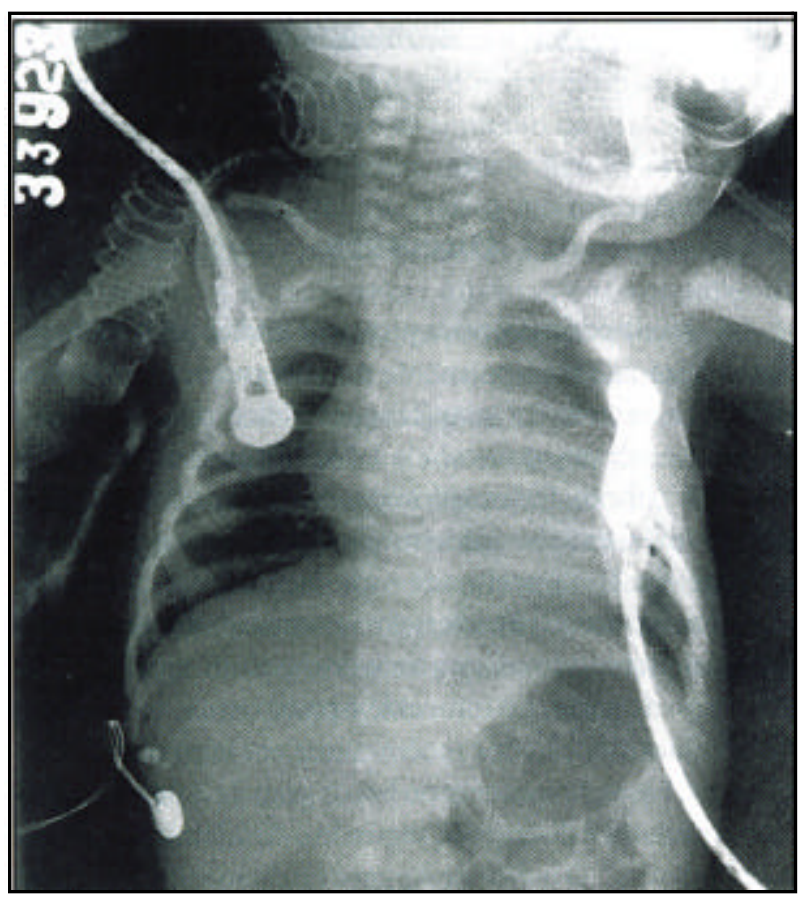

Fig. 1 - Radiografia de tórax de um RN, mostrando aumento da área cardíaca. 


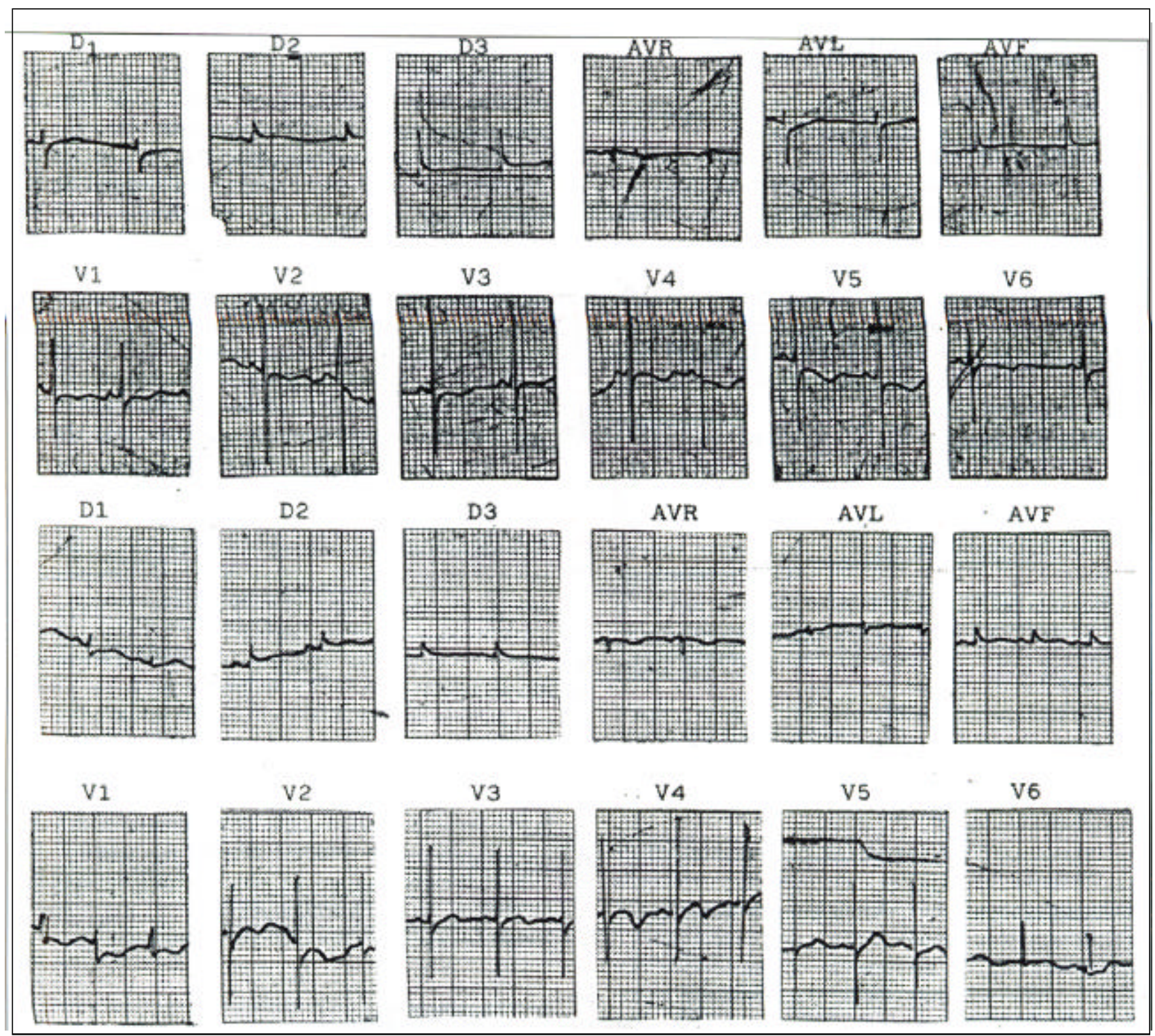

Fig. 2 - ECG com alterações difusas de repolarização ventricular (ondas $\mathrm{T}$ negativas em $\mathrm{V}_{4}, \mathrm{~V}_{5}$ e $\mathrm{V}_{6}$ ). E achatadas e na evolução dias depois com melhora acentuada.

\section{Discussão}

A asfixia perinatal pode provocar alterações graves em vários órgãos, principalmente no sistema nervoso, rins, pulmões e coração ${ }^{4}$. Complicações observadas em nosso estudo encontram-se na tabela I. Nos últimos anos, vários trabalhos ${ }^{4-8}$ foram formulados com o objetivo de relacionar as possíveis alterações encontradas em vários órgãos, causadas pela asfixia perinatal com marcadores clínicos e bioquímicos, como $\mathrm{pH}$ arterial e anormalidades do cordão umbilical, líquido amniótico tinto de mecônio, apresentação fetal e tipo de parto, e o índice de Apgar.

A classificação dos recém-natos em hipoxêmicos ou asfíxicos, baseada somente no baixo grau de Apgar nos primeiros minutos é discutível, segundo a Academia Americana de Pediatria ${ }^{5}$. Oíndice de Apgaré um bom indicativo para condutas utilizadas em sala de parto, mas não pode ser rela- cionado com possíveis evidências de disfunções multiorgânicas, já que pode ser influenciado por vários fatores maternos e fetais. Entretanto, Martin-Ancel e $\mathrm{col}^{4}$ realizaram estudo para classificar em asfíxicos, recém-natos que apresentavam pelo menos três dos vários parâmetros: $\mathrm{pH}$ sangüíneo ou do cordão umbilical $<7,2$, Apgar $<4$ no $1^{\circ}$ min e/ou $<7$ no $5^{\circ} \mathrm{min}$, e necessidade de mais de $1 \mathrm{~min}$ de ventilação com pressão positiva antes da sustentação da respiração. Verificaram que o baixo Apgar no $1^{\circ}$ e no $5^{\circ} \mathrm{min}$ foi o único marcador perinatal de falência associado com o número total de órgãos afetados em cada criança. Tivemos vários casos com complicações neurológicas, principalmente, convulsões. Perlman e col observaram freqüência de casos semelhantes à nossa ${ }^{6}$.

No nosso material, tentou-se correlacionar o baixo grau de Apgar e o pH sangüíneo com as alterações clínicocardiológicas. A gasometria mostrou baixos níveis de $\mathrm{pH}$, 


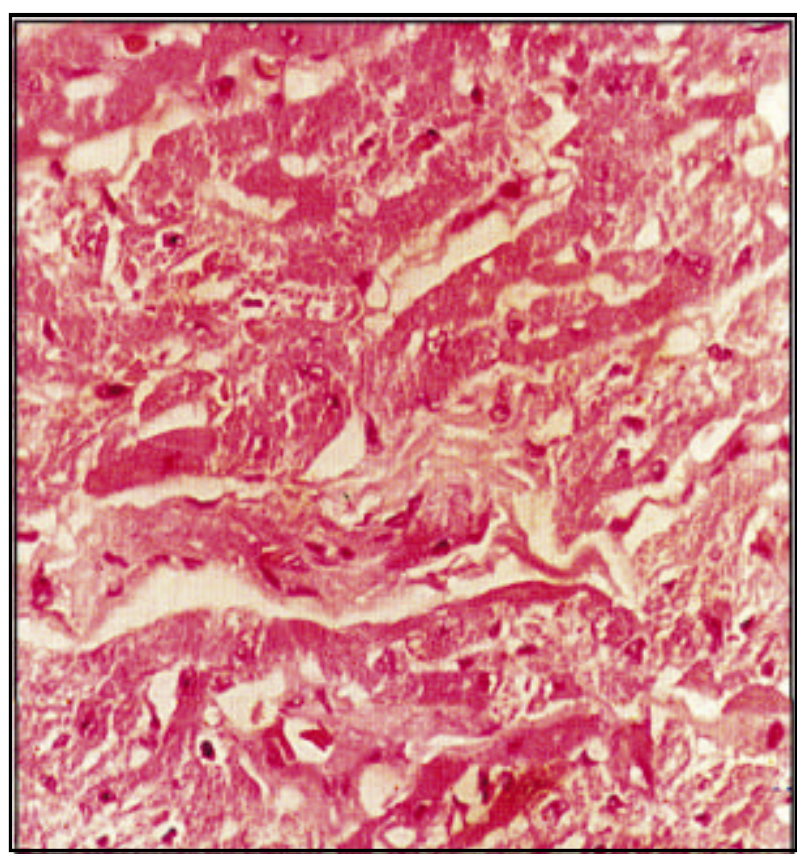

Fig. 3 - Microscopia do coração mostrando edema interfibrilar, vacuolização e perda da estriação das fibras (H.E $100 \mathrm{X}$ ).

principalmente nos bebês que foram reanimados (46\%), entretanto, muitos desses evoluíram com quadro clínico ECG e ecocardiograma normais.

Asfixia neonatal com isquemia miocárdica foi descrita pela primeira vez em 1972 por Rowe e Hoffman ${ }^{7}$. Alguns desses casos apresentavam também regurgitação tricúspide, causada pelo aumento de pressão na artéria pulmonar por vasoconstrição secundária à hipóxia, ocasionando um maior trabalho de VD, e acarretando consumo acentuado de oxigênio $\left(\mathrm{O}_{2}\right)$ pelo miocárdio ventricular, diminuição da perfusão subendocárdica, isquemia, falência ventricular direita e regurgitação tricúspide.

Em nossa casuística, foram observados alguns casos de regurgitação tricúspide, sendo em um, fatal. Algumas hipóteses para justificar casos de insuficiência tricúspide transitória, detectada no período neonatal, são descritas na literatura ${ }^{2,8,9}$. Para uns, o fechamento precoce do canal arterial e foramen ovale aumentaria a pressão no VD, facilitando a regurgitação. Para outros, o retardo no crescimento do folheto septal da tricúspide seria a causa primária desse fato. Processos isquêmicos prolongados podem acarretar infarto de estruturas do aparelho valvar, como ocorrido em nosso caso e de outros autores ${ }^{2,9,10}$.

As alterações eletrocardiográficas mais freqüentes por nós observadas e por vários autores foram as modificações do segmento ST e onda T. Usando cintilografia com tálio, Finley e col mostraram que essas alterações eletrocardiográficas regridem após alguns meses ${ }^{11}$. A diminuição ou ausência de captação do tálio pelo miocárdio isquêmico é

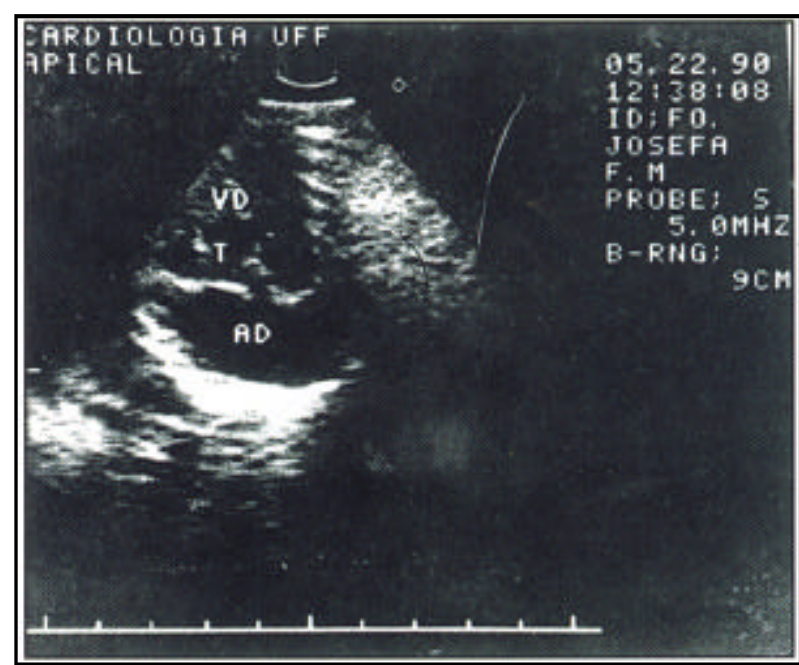

Fig. 4 - Ecocardiograma bidimensional mostrando rotura da cordoalha da válvula tricúspide.

provavelmente conseqüente a alterações da bomba de sódio-potássio através da membrana das miofibrilas. Constatamos os valores elevados de isoenzimas CPK-MB em alguns bebês com alterações eletrocardiográficas importantes, dados, também, obtidos por Bucciarelli e col ${ }^{8}$.

Em nossa casuística, 22\% apresentaram PCA, fato também relatado por Van Bele Walther ${ }^{12}$. Em estudo experimental, provocando hipóxia crônica em porcos recém-nascidos, Plumkett e col verificaram que o miocárdio sofreu inúmeras alterações celulares metabólicas e bioquímicas, mas apenas um animal apresentou $\mathrm{PCA}^{13}$. Em alguns de nossos casos, o ecocardiograma mostrou a disfunção ventricular, entretanto, outros autores observaram baixos índices sistólicos com maior freqüência ${ }^{12}$.

Estudos experimentais mostram que o miocárdio de animal recém-nascido e, mais ainda, de feto é resistente aos efeitos da hipóxia. Estes mecanismos de adaptação são conseqüentes aos níveis elevados de fosfatos ricos em energia, glicogênio, havendo aumento da glicogenólise. Nesta situação, o desempenho miocárdico fica na dependência da glicose anaeróbica. Assim, modificações nos níveis de glicogênio cardíaco levam a alterações eletrocardiográficas semelhantes àquelas encontradas na isquemia miocárdica. Em etapas precoces, a reversibilidade destes fenômenos pode ser ainda conseguida com a administração de $\mathrm{O}_{2}{ }^{14}$. Parece-nos que as diferenças no quadro clínico dos bebês asfíxicos e sua evolução dependem da capacidade adaptativa do miocárdio de cada um.

Concluímos que a maioria dos bebês teve evolução favorável, mesmo aqueles que tiveram acidemia importante. Dos que evoluíram para óbito, foram observadas lesões histológicas mais graves nos que sofreram processo anóxico por tempo prolongado. 


\section{Referências}

1. Herdy GVH, Miranda GR, Lopes VGS et al - Complicações cardíacas da asfixia perinatal. Arq Bras Pediatria 1994, 1: 131-6.

2. Pinto CAM, Herdy GVH, Ferrari AH et al - Insuficiência tricúspide severa por infarto do músculo papilar anterior do VD secundário à hipóxia perinatal. Arq Bras Cardiol 1992, 58: 475-8.

3. Freyman R, Dallfez HC - Transient tricuspid incompetence in newborn. Eur J Cardiol 1975, 2: 467-71.

4. Martin-Ancel A, Alix AG, Gayá F et al - Multiple organ involvement in perinatal asphyxia. J Pediatrics 1995, 127: 786-93.

5. American Academy of Pediatrics - Use and abuse of the Apgar score. Pediatrics 1996, 98: 141-2.

6. Perlman JM, Risser R - Can asphyxiated infants at risk for neonatal seizures be rapidly identified by current high - risk markers? Pediatrics 1996, 97: 456-62.

7. Rowe RD, Hoffman T - Transient myocardial ischemia of the newborn infant: a of a severe cardiorespiratory distress in full-term infants. J Pediatrics 1972, 81: 243-50.

8. Bucciarelli RL, Hoffman T-Transiend myocardial ischemia of the newborn infant: a of a severe cardiorespiratory distress in full-term infantis. J Pediatrics 1972, 81: 243-50.

9. Donnely WH, Bucciarelli RL, Nelson RM - Ischemic papilary muscles necrosis in stressed newborn infants. Pediatrics 1980, 96: 295-300.

10. Alkalay AL, Ferry DA, Pepkowitz SH et al - Critical Tricuspid insufficiency due to papillary muscle rupture. Am J Dis Child. A988; 142: 753-5.

11. Finley JP, Hownan G, Gilday DL - Transient myocardial Ischemia of the newborn infant demostrated by tallium myocardial imaging. J Pediatr 1979; 94 : 263-70.

12. Van BelF, Walter FJ - Myocardial dysfunction on and cerebral blood flow velocity following birth asphyxia. Acta Paed Scand 1990; 79: 756-62.

13. Plunkett MD, Hendry PJ, Anstadt MP et al - Chronic hypoxia induces adaptative metabolic changes in neonatal myocardium. J Thorac Cardiovasc Surgery 1996, 112: 8-13.

14. Hoerter JA, Opie LH - Perinatal changes in Glycolytic function in response to hypoxia in the incubated or perfused rat heart. Biol Neoate 1978; 33: 144-61. 